\title{
Apport de l'observation satellitaire à I'hydrologie continentale
}

\author{
Sylvain Biancamaria ${ }^{1}$, Yann Kerr ${ }^{2}$ \\ 1 Laboratoire d'études en géophysique et océanographie spatiales, \\ Université Paul-Sabatier / Cnes / CNRS / IRD, Toulouse \\ 2 Centre d'études spatiales de la biosphère, Université Paul-Sabatier / \\ Cnes / CNRS / IRD, Toulouse \\ sylvain.biancamaria@legos.obs-mip.fr
}

\section{Résumé}

Depuis plusieurs décennies, un nombre croissant de satellites observe la variabilité spatio-temporelle de plusieurs compartiments des eaux continentales (eaux libres, manteau neigeux, humidité du sol...). Ces observations ont permis de mieux comprendre les échanges d'eau au sein des bassins fluviaux et le couplage avec l'atmosphère et les océans. Elles ont aussi contribué à améliorer les prévisions d'inondation, la prévention de risques naturels et le suivi de l'évolution géomorphologique des zones en eau. Les futures missions spatiales, en améliorant la précision et la récurrence des mesures, deviendront un outil incontournable pour gérer les ressources en eau.

\section{Abstract}

\section{Contribution \\ of satellite observations to continental hydrology}

Over the last decades, an increasing number of satellite missions has helped to observe the spatial and temporal variabilities of continental waters (surface water, surface snow, soil moisture, etc.). These observations helped to better understand water fluxes within river basins and their coupling with the atmosphere and oceans. They also contributed to improve flood forecasting, prevent natural hazards and track geomorphological changes in water bodies. Future satellite missions, with improved measurement accuracy and temporal sampling, will become essential tools for water resources management.
D ès le début de l'ère spatiale, après le lancement de Spoutnik-1 en 1957, un certain nombre de satellites se sont attachés à observer les surfaces continentales, initialement avec des objectifs militaires d'espionnage, mais aussi, très rapidement, en vue d'applications à caractère météorologique (séries de satellites météorologiques de la National Oceanic and Atmospheric Administration, Noaa) ou cartographique (Heat Capacity Mapping Mission, Landsat, Spot...). De fait, ce nouveau moyen d'observation s'est rapidement avéré être une source précieuse d'informations pour la science. Par exemple, comme le rappellent Lettenmaier et al. (2015), les premiers radiomètres, qui mesurent le rayonnement électromagnétique émis par la Terre dans plusieurs longueurs d'onde, ont été embarqués sur Explorer-7 (lancé en 1959), puis sur la série de satellites Tiros (Television Infrared Observation Satellite, le premier ayant été lancé en 1960). Ces données ont permis, outre leurs applications en météorologie, les premières études du bilan radiatif terrestre, essentiel pour l'étude du climat et de son évolution.

En particulier, durant cette période, les premiers satellites qui ont photographié la Terre sont les satellites espions (comme le programme américain Corona, de 1959 à 1972), qui embarquaient des appareils photo, ceux-ci éjectant les pellicules photographiques utilisées. La capsule contenant les pellicules était alors récupérée (dans l'atmosphère par des avions militaires ou au sol), puis les pellicules développées et analysées. Ces données étaient évidemment classées et ne couvraient que certaines zones stratégiques (selon le pays qui exploitait ces données). Mais l'amélioration des capteurs et le développement de systèmes automatiques de téléchargement au sol des télémesures émises par les satellites ont rapidement permis le développement de missions civiles d'observation de la Terre (initialement dans le visible et l'infrarouge notamment), comme le programme américain Landsat (Landsat-1 a été lancé en 1972 ; le dernier en date, Landsat-8, en 2013) ou le programme français Spot (Satellite pour l'observation de la Terre ; Spot-1 a été mis en orbite en 1986 et Spot-7 en 2014). C'est notamment grâce au programme Landsat, dont les données ont été mises à disposition de la communauté scientifique, que les données satellitaires se sont imposées comme des compléments essentiels des réseaux d'observation au sol ou aéroportés pour l'étude de l'hydrologie continentale. Ces observations satellitaires ont, par exemple, permis d'observer les variations de l'étendue du manteau neigeux, de la superficie de lacs, des réservoirs et des plaines d'inondation à des échelles spatiales sans précédent et ont apporté des observations inédites sur des bassins fluviaux peu (voire pas) jaugés.

Depuis les années 1970 et ces premiers satellites, un nombre important de missions, embarquant une grande variété de capteurs, ont été lancées par les différentes agences spatiales. Elles ont permis de grandes avancées 
scientifiques, notamment dans l'observation et la connaissance de la partie continentale du cycle de l'eau.

L'intérêt des données satellitaires est multiple. Le satellite permet une couverture spatiale assez large (souvent mondiale pour les missions en orbite polaire) des surfaces continentales, de façon répétitive (le satellite repasse au-dessus du même point à intervalles de temps réguliers), les mesures sont effectuées par le même instrument, étalonné de la même manière. Ces mesures sont ensuite traitées avec les mêmes algorithmes, quel que soit le point du globe observé (contrairement aux réseaux d'observations in situ qui dépendent des politiques nationales, voire locales), ce qui est particulièrement intéressant pour des bassins fluviaux transfrontaliers (recouvrant plusieurs pays) et/ou peu observés. De plus, la mise à disposition gratuite, au moins pour une utilisation scientifique, des données satellitaires par certaines agences spatiales a indubitablement favorisé leur utilisation.

\section{Observations satellitaires des différents compartiments des eaux continentales}

L'hydrologie continentale porte sur l'étude des flux d'eau sur les continents (figure 1), aussi bien en surface (rivières, lacs, stock de neige) que dans les sols (zone non saturée, aquifères profonds). Aujourd'hui, plus d'une vingtaine de satellites embarquent des instruments permettant d'observer (plus ou moins directement) un ou plusieurs compartiments de cette partie continentale du cycle de l'eau. Le tableau 1 fournit une vision d'ensemble de ces missions et des variables observées. L'idéal serait de pouvoir mesurer directement les volumes d'eau et leur variation spatio-temporelle, mais cela est rarement possible. Les compartiments les mieux observés sont les eaux de surface, à savoir : le couvert neigeux (c'est-à-dire la superficie), l'humidité du sol, les eaux à surface libre (lacs, réservoirs, rivières et fleuves). Les aquifères (de surface ou en profondeur) ne peuvent pas être observés directement par des satellites, mais ils peuvent être étudiés indirectement sous certaines conditions.

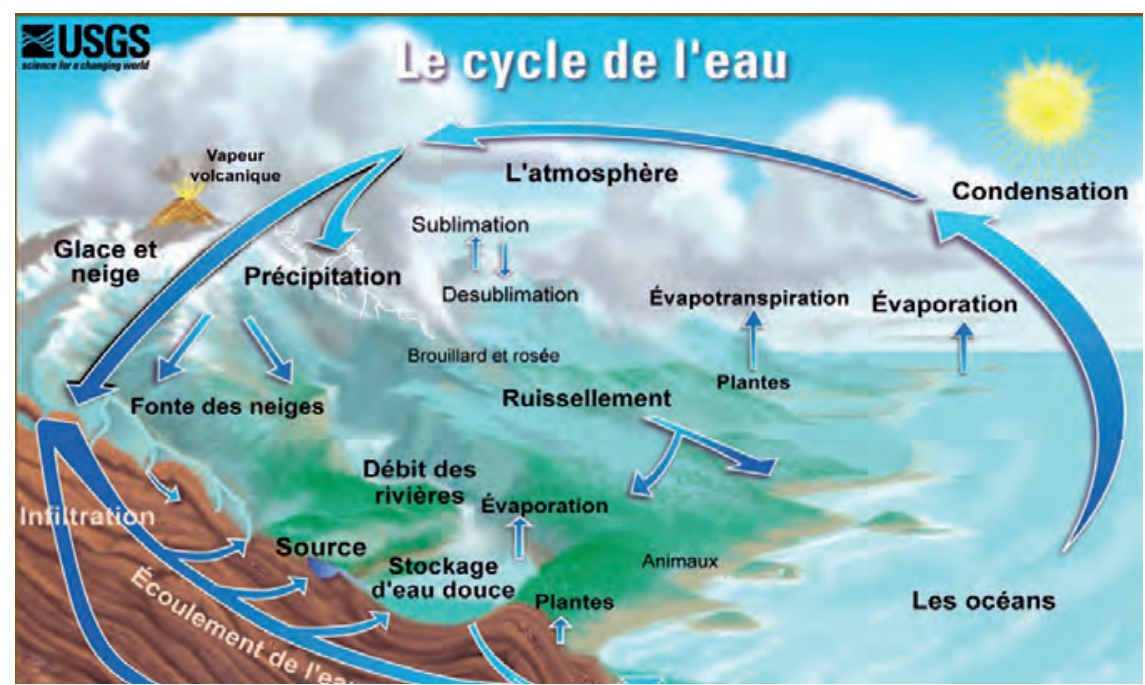

Figure 1. Le cycle de l'eau. D'après une illustration de J. M. Evans et H. Perlman, USGS Georgia Water Science Center, traduction en français par Monika Michel, Agence de l'eau Artois-Picardie, http://ga.water.usgs.gov/edu/watercyclefrenchhi.html.

Tableau 1. Variables utilisées en hydrologie continentale et missions satellitaires (avec leurs caractéristiques) disponibles en 2016 pour les observer (inventaire non exhaustif et ne regroupant que des données disponibles en accès libre au moins pour les données d'archive et pour certaines résolutions).

\begin{tabular}{|c|c|c|c|c|c|}
\hline Variable & $\begin{array}{l}\text { Mission } \\
\text { satellitaire }\end{array}$ & $\begin{array}{l}\text { Types } \\
\text { d'instrument }\end{array}$ & $\begin{array}{l}\text { Échantillonnage } \\
\text { spatial }\end{array}$ & $\begin{array}{l}\text { Échantillonnage } \\
\text { temporel }\end{array}$ & $\begin{array}{l}\text { Délai de mise } \\
\text { à disposition } \\
\text { de la donnée }\end{array}$ \\
\hline Précipitations & $\begin{array}{l}\text { Constellation } \\
\text { GPM } \\
\text { et satellites } \\
\text { géostationnaires }\end{array}$ & $\begin{array}{l}\text { Radiomètres, } \\
\text { radar... }\end{array}$ & 10 à 100 km & 1 heure à 1 jour & $\begin{array}{l}\text { Quelques heures } \\
\text { à } 1 \text { mois }\end{array}$ \\
\hline $\begin{array}{l}\text { Pourcentage } \\
\text { de zone en eau }\end{array}$ & $\begin{array}{l}\text { GCOM-W1 } \\
\text { (AMSR2), Smos }\end{array}$ & $\begin{array}{l}\text { Radiomètres } \\
\text { micro-ondes }\end{array}$ & $25 \mathrm{~km}$ & $\sim 1$ jour & $\sim 1$ jour \\
\hline \multirow[t]{2}{*}{$\begin{array}{l}\text { Étendue d'eau } \\
\text { (et de neige) }\end{array}$} & $\begin{array}{l}\text { Aqua et Terra } \\
\text { (Modis), } \\
\text { Landsat-7 et } 8 \text {, } \\
\text { Spot- } 6 \text { et } 7 \text {, } \\
\text { Sentinel-2A et } 3 A\end{array}$ & $\begin{array}{l}\text { Radiomètres } \\
\text { visible } \mathrm{x} \\
\text { visible }\end{array}$ & ～ 1 à 500 m & 1 jour à $\sim 15$ jours & $\begin{array}{l}\text { Quelques heures } \\
\text { à quelques jours }\end{array}$ \\
\hline & $\begin{array}{l}\text { TerraSAR-X, } \\
\text { Tandem-X, } \\
\text { Radarsat-2, } \\
\text { Sentinel-1 }\end{array}$ & Radar & 1 à 40 m & Quelques jours & Quelques heures \\
\hline \multirow[t]{2}{*}{ Cote d'eau } & $\begin{array}{l}\text { Cryosat-2, } \\
\text { HY-2A, Jason-2 } \\
\text { et 3, Saral, } \\
\text { Sentinel-3A }\end{array}$ & Altimètres & Ponctuel & $\begin{array}{l}10 \text { à } 35 \text { jours } \\
\text { radar nadirs }\end{array}$ & $\begin{array}{l}<1 \text { jour à } 1 \text { mois } \\
\text { (série Jason) }\end{array}$ \\
\hline & $\begin{array}{l}\text { lcesat-2 } \\
\text { (lancement } \\
\text { prévu en 2017) }\end{array}$ & Lidar & Ponctuel & 91 jours & 1 mois \\
\hline $\begin{array}{l}\text { Humidité } \\
\text { des premiers } \\
\text { centimètres } \\
\text { du sol et zone } \\
\text { racinaire }\end{array}$ & Smos, Smap & $\begin{array}{l}\text { Radiomètres } \\
\text { bande L }\end{array}$ & $\sim 40 \mathrm{~km}$ & $\sim 1$ jour & $\begin{array}{l}<3 \text { h à quelques } \\
\text { jours }\end{array}$ \\
\hline $\begin{array}{l}\text { Variation } \\
\text { du stock } \\
\text { d'eau total }\end{array}$ & Grace & $\begin{array}{l}\text { Distance entre } \\
\text { deux satellites }\end{array}$ & $\sim 300 \mathrm{~km}$ & 1 mois & Quelques mois \\
\hline
\end{tabular}

Pour ce qui est de l'observation des surfaces enneigées et englacées (qui peuvent jouer un rôle très important sur l'hydrologie de certains bassins fluviaux), on se référera à Rabatel et al.
(2017), ce numéro. Mais il importe de rappeler que l'observation des surfaces enneigées et englacées est très importante pour étudier le cycle de l'eau continental. 
L'humidité superficielle du sol est difficile à mesurer, car il faut être capable de pénétrer l'atmosphère et le couvert végétal avec un mode d'observation qui soit sensible au contenu en eau et peu affecté par les autres milieux traversés. Il importe également d'intégrer la mesure sur une couche de sol afin de s'affranchir des variations rapides liées notamment à l'évolution des conditions météorologiques de surface comme le vent. Avant 2009, aucune mission satellitaire n'avait été conçue pour observer spécifiquement cette variable. Certaines mesures optiques ou micro-ondes avaient pu être, a posteriori, plus ou moins reliées à l'humidité des sols (pas forcément de manière quantitative), mais ces instruments n'observaient pas dans la gamme de longueurs d'onde la plus adaptée. La meilleure option repose sur l'utilisation des micro-ondes à basse fréquence et plus particulièrement l'utilisation de la bande L (20 cm) (Kerr, 2007). En effet, pour les instruments actifs (radar et diffusiomètre), le rapport signal sur bruit est plus faible et les effets liés à la rugosité du sol et à la végétation sont plus importants. La mission Smos (Soil Moisture and Ocean Salinity, voir encadré 1) a été lancée en novembre 2009 et vise à obtenir une cartographie régulière (avec plus de deux acquisitions tous les trois jours) de l'humidité superficielle sur les surfaces continentales (Kerr et al., 2016). Toujours opérationnelle, la mission Smos a été suivie par la mission Smap lancée en 2015 (Entekhabi et al., 2010). Elle repose sur un radiomètre interférométrique opérant en bande L (1 400-1 $427 \mathrm{MHz}$ ) dont la profondeur de pénétration dans les sols est de 2 à $5 \mathrm{~cm}$ en moyenne (Waldteufel et al., 2012). Le capteur, par sa capacité multiangulaire, permet de séparer les contributions émanant du couvert végétal de celles du sol (Kerr et al., 2016). Smos permet ainsi d'observer l'humidité des sols, même sous une végétation

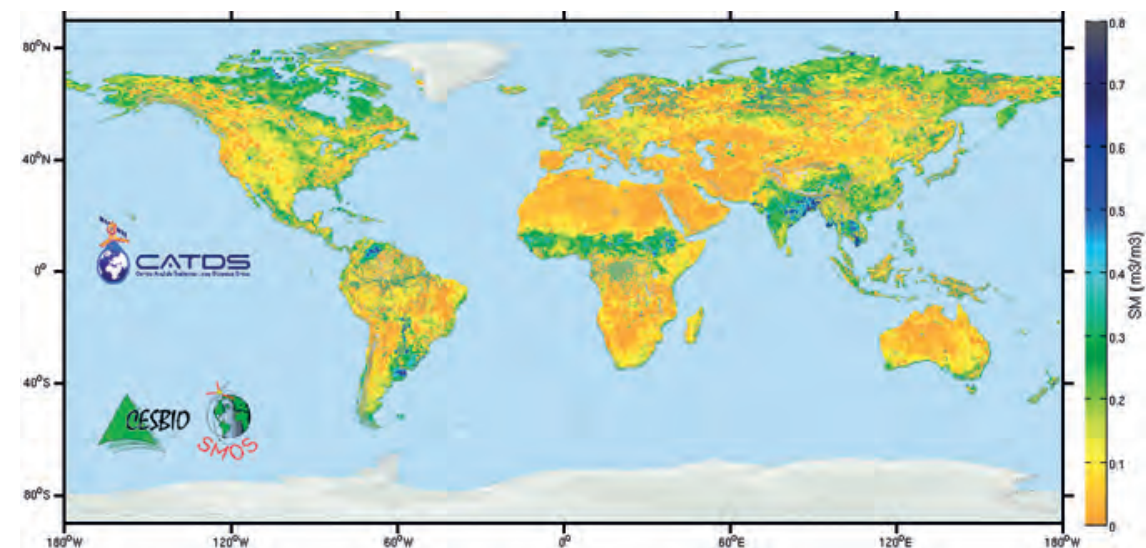

Figure 2. Carte mensuelle d'humidité superficielle $\left(\mathrm{m}^{3} / \mathrm{m}^{3}\right)$ pour le mois d'août 2014 obtenue par la mission Smos.

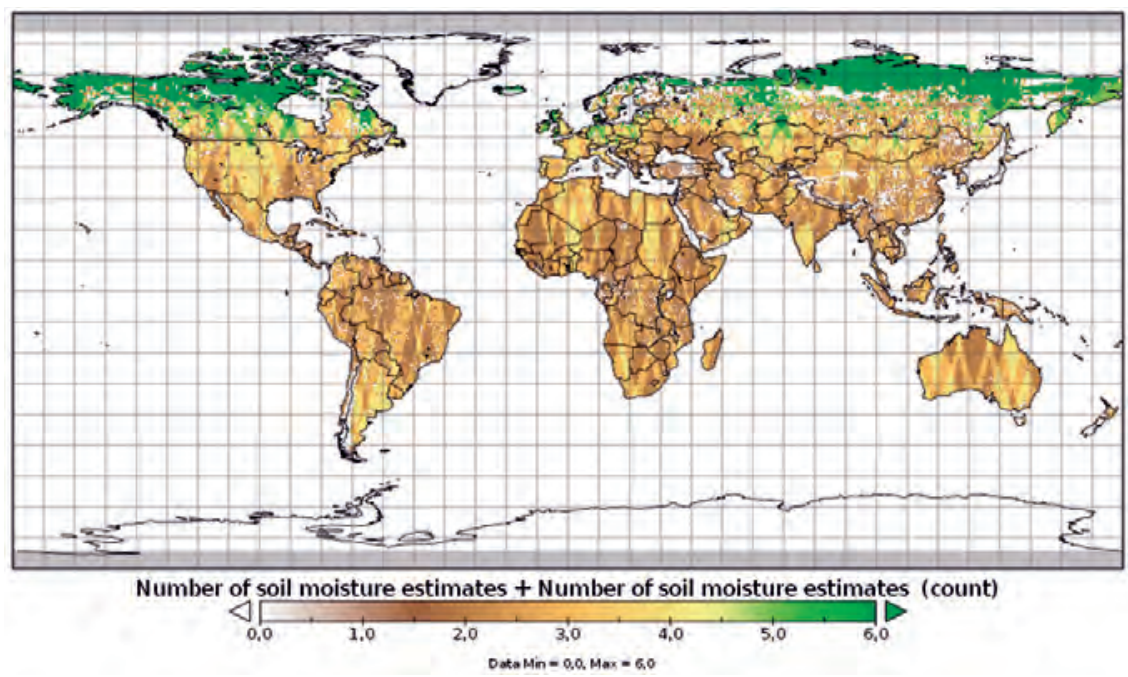

Figure 3. Nombre d'inversions d'humidité superficielle obtenues par Smos sur une période de 3 jours (du $1^{\text {er }}$ au 3 août 2015). Les trous d'observation correspondent aux zones de forte topographie, recouvertes de neige ou affectées par des interférences parasites avec des appareils au sol pour lesquelles il n'y a pas d'inversion. relativement dense, et ce de façon absolue (contrairement aux indices qualitatifs fournis par d'autres instruments) et homogène (l'algorithme ne fonctionne pas pixel par pixel) sur l'ensemble du globe (figure 2). Les mesures sont assez fréquentes (figure 3) pour suivre les événements

\section{La mission Smos}

La mission Smos (Soil Moisture and Ocean Salinity, lancée le 2 novembre 2009) est la première mission en bande L (1 400-1 $427 \mathrm{MHz}$ ) utilisant un concept interférométrique spatialisé fournissant des données d'humidité et de salinité sur l'ensemble du globe. Grâce au concept interférométrique, il est possible d'avoir des résolutions spatiale et radiométrique adaptées pour le suivi de la salinité des océans et de I'humidité superficielle des sols $(2,5 \mathrm{~K}$ pour les océans et empreinte à $3 \mathrm{~dB}$ de $43 \mathrm{~km}$ ). Le système se caractérise par des acquisitions en pleine polarisation multiangulaires permettant d'améliorer la sensibilité et surtout permettant de séparer différentes contributions au signal (par exemple, les contributions du couvert végétal et du sol). Grâce à ces caractéristiques, l'ensemble du globe est observé plus de deux fois tous les trois jours, les données étant disponibles en temps quasi réel (températures de brillance et humidité moins de $3 \mathrm{~h}$ après acquisition) et assimilé dans les modèles de prévision du temps (ECMWF). Cela a généré de multiples applications en hydrologie (estimation des pluies, risque $d^{\prime}$ inondations, etc.) et dans d'autres domaines (suivi des vents forts sur les océans, des glaces de mer fines, du gel/dégel des sols, de la biomasse des forêts). Deux autres concepts ont vu le jour depuis (antenne push broom avec Aquarius (2011-2015) et antenne déployable avec Smap, lancé en janvier 2015). Le problème actuel est d'assurer la continuité de ces observations compte tenu de leur valeur scientifique et de leurs applications.

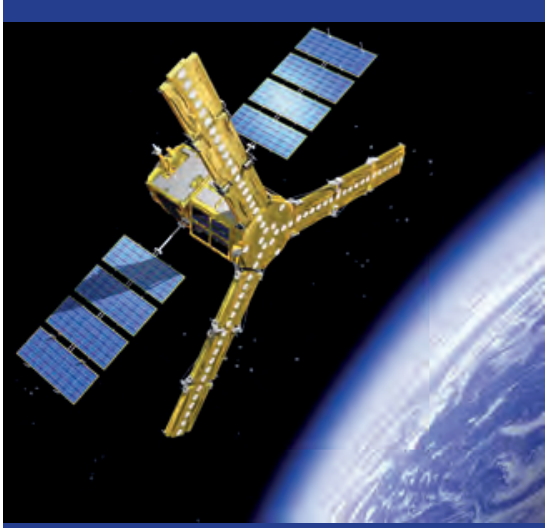

Vue d'artiste du satellite Smos lancé le 2 novembre 2009. Source : Cesbio-Mira. 
hydrologiques (assèchement des sols, épisodes pluvieux) de façon précise. Les principales limitations de Smos sont relatives à sa résolution spatiale (40 km échantillonnés à 15 ou $25 \mathrm{~km}$ ) et sa profondeur de pénétration. Pour la première limitation, des approches de désagrégation ont été mises au point, qui permettent d'obtenir des résolutions kilométriques ou hectométriques (Merlin et al., 2013), favorisant des applications en agronomie et gestion de la ressource en eau. Pour la seconde, différentes techniques ont été développées pour estimer l'humidité dans la zone racinaire par assimilation dans des modèles de l'humidité de surface. Cette information est ensuite utilisée afin d'obtenir des indicateurs de sécheresse ou de sécurité alimentaire. Elle est aussi utilisée afin d'établir des cartes de risques d'inondation. L'humidité des sols étant un traceur des pluies, il est également possible de corriger les estimations de précipitations liquides obtenues par satellite en assimilant l'humidité superficielle dans des modèles hydrologiques, permettant ainsi d'obtenir une estimation des précipitations plus réaliste (Brocca et al., 2016). D'autres travaux (Schwank et al., 2015) montrent qu'il est possible d'obtenir la densité de la neige. Une équipe à l'Institut météorologique finlandais travaille par ailleurs sur le suivi au niveau mondial du gel et du dégel (Rautiainen et al., 2016). Les données d'humidité superficielle Smos sont par ailleurs disponibles en temps quasi réel, tout comme les températures de brillance pour des applications en prévision météorologique bien sûr, mais aussi pour la prévention des risques (inondations en particulier).

En ce qui concerne les eaux à surface libre, leurs étendues et leurs variations sont estimées à l'aide de données de radiomètres opérant dans le visible et l'infrarouge (imagerie optique), de radiomètres mesurant le rayonnement micro-ondes émis par les surfaces continentales ou bien à l'aide de radars. L'intérêt des missions d'imagerie optique est leur large emprise au sol (ou fauchée), le nombre important de fréquences électromagnétiques échantillonnées (en général du visible à l'infrarouge thermique) et la résolution spatiales des images (de plusieurs centaines de mètres à quelques centimètres pour certaines missions militaires et commerciales). Les missions Landsat-8 (lancé par la Nasa et l'USGS ${ }^{1}$ en 2013) et Sentinel-2 (dont le premier satellite a été lancé en 2015 par l'ESA ${ }^{2}$ ) permettent une couverture globale des surfaces continentales avec un temps de revisite de 16 jours et une résolution de $30 \mathrm{~m}$ pour Landsat-8, un temps de revisite de 5 jours et une résolution de 10 à $20 \mathrm{~m}$ pour Sentinel-2. Les images acquises dans plusieurs fréquences électromagnétiques permettent de calculer un ensemble de produits très variés : masque des eaux à surface libre, masque de nuages et de leur ombre portée au sol, classification de sol, étendue des zones enneigées, indice de croissance de la végétation... Le problème des systèmes optiques est l'absence de données exploitables en présence de nuages, ce qui est fréquent, notamment en période de mousson pour les bassins tropicaux. Une alternative est l'utilisation de radar (voir le tableau 1), les nuages étant transparents aux fréquences utilisées par ces capteurs actifs. Il y a cependant moins de missions en orbite, avec des résolutions plus variables et une utilisation potentiellement plus complexe. Les masques d'eau (provenant des images optiques ou radars) ont permis de dresser des inventaires plus ou moins globaux des lacs et des réseaux de drainage qui sont très importants pour les modèles hydrologiques et hydrauliques. Leurs variations temporelles permettent d'étudier la géomorphologie des fleuves et le mouvement de leur lit mineur, ainsi que la dynamique des lacs et réservoirs d'eau.

Cependant, si l'imagerie optique permet de calculer la superficie des zones d'eau et ses variations dans le temps, il est nécessaire de disposer aussi des variations de hauteur d'eau pour obtenir des variations de volume (et donc de masse) d'eau, qui est une variable physique pertinente pour faire un bilan hydrologique. Ceci peut être fait en combinant ces masques avec des modèles numériques de terrain (MNT), mais ceux-ci doivent être suffisamment précis pour être compatibles avec les masques d'eau et les variations de hauteur à observer. Les MNT globaux disponibles en libre accès ayant des erreurs verticales de l'ordre de la dizaine de mètres ou plus, ils ne peuvent être utilisés que pour des zones d'eau importantes ayant des variations de hauteur de plusieurs mètres entre les basses et les hautes eaux. Des MNT locaux plus précis (précision verticale absolue métrique, voire décimétrique) peuvent être utilisés lorsqu'ils sont disponibles, ce qui n'est pas souvent le cas à l'échelle globale. Une alternative consiste à utiliser l'altimétrie satellitaire (tableau 1, voir encadré 2).

\section{L'altimétrie par satellite}

L'altimétrie nadir satellitaire observe

les zones d'eau à surface libre (fleuves,

rivières, lacs et réservoirs). Elle permet

de mesurer la distance entre la surface

de la zone d'eau observée et une

surface de référence (ellipsoïde ou

géoïde). Cette distance est appelée

" cote d'eau » par les hydrologues. Par

contre, l'altimétrie nadir ne permet pas

de calculer la profondeur d'eau,

$c^{\prime}$ est-à-dire la distance entre la surface et le fond du fleuve, rivière, lac ou réservoir. Pour avoir accès à cette profondeur, il faut disposer d'une estimation a priori de la bathymétrie de la zone d'eau considérée. II existe deux types d'instrument d'altimétrie nadir : les altimètres radars et les lidars. Les lidars sont plus précis, mais ne " voient» pas à travers les nuages, contrairement aux radars.

L'altimétrie nadir fournit des mesures seulement le long de la trace du satellite à sa verticale (son nadir), ce qui limite énormément le nombre de cibles observées. La distance entre deux traces varie en fonction de l'orbite du satellite: plus l'échantillonnage temporel est important, moins l'échantillonnage spatial est dense. Par exemple, la mission Jason-3 (lancée le 17 janvier 2016) a une période de répétitivité de 10 jours et une distance entre deux traces à l'équateur de $315 \mathrm{~km}$, tandis que la mission Sentinel-3A (lancée le 16 février 2016) a une répétitivité de 27 jours, mais une distance entre deux traces à l'équateur de $104 \mathrm{~km}$.

Par contre, la future mission spatiale Swot (Surface Water and Ocean Topography), développée conjointement par la Nasa, le Cnes, la CSA/ACS (Canadian Space Agency/Agence spatiale canadienne) et la UK Space Agency qui sera une mission d'altimétrie radar à large fauchée et dont le lancement est prévu pour 2021, fournira des images de cotes d'eau sur deux fauchées de $50 \mathrm{~km}$ de large chacune.

À l'heure actuelle, seuls des altimètres à visée nadir sont disponibles : ils fournissent des cotes d'eau (distance entre la surface d'eau et une surface de référence, géoïde ou ellipsoïde) le long de la trace du satellite, mais ne permettent pas d'échantillonner perpendiculairement à la trace du satellite et d'obtenir des « images » de cotes d'eau. Ils ne peuvent donc pas

1. United States Geological Survey.

2. European Space Agency, Agence spatiale européenne. 
observer l'ensemble des zones d'eau à surface libre. Les altimètres radars au nadir sont les plus courants et sont utilisés depuis plus de 20 ans sur les surfaces continentales. Ils ont un temps de revisite qui peut varier de 10 à 35 jours en fonction des missions et ont permis d'observer des variations de cote d'eau sur un grand nombre de grands fleuves, lacs et réservoirs. Ces mesures commencent à être de plus en plus utilisées sur des rivières et fleuves de taille moyenne (200 m de large et moins), mais les précisions verticales sont très dépendantes de la topographie environnante et, pour des rivières de petite taille, une répétitivité de 10 jours devient limitante. Cependant, combinées avec des masques d'eau, ces données ont permis de fournir des variations de volume de grands lacs et réservoirs (Crétaux et al., 2016 et figure 4), voire de débit de fleuves lorsque des données in situ sont disponibles au moins sur une courte période, ce qui s'est avéré une source d'information importante pour les bassins non ou peu jaugés.

Depuis 2002, la mission Grace (Gravity Recovery and Climate Experiment; voir encadré 3) mesure le champ de gravité terrestre et ses très faibles variations temporelles. Ces données permettent d'estimer les changements de masses d'eau dans les couches superficielles de la Terre, à l'échelle mensuelle et avec une résolution spatiale de l'ordre de $300 \mathrm{~km}$ (voir figure 5). Ces mesures sont donc évidemment utilisées à l'échelle globale ou pour des bassins de grande taille. La mission Grace permet d'observer les variations de la masse d'eau intégrée sur toute la colonne de sol, c'est-à-dire depuis la surface (zones d'eau à surface libre,

\section{La gravimétrie par satellite}

La mission Grace (Gravity Recovery and Climate Experiment, lancée le 17 mars 2002), résultant d'une collaboration entre la Nasa et le DLR (Deutsche Raumfahrt), est composée de deux satellites volants en tandem et séparés par une distance de $220 \mathrm{~km}$. Les variations de distance entre les deux satellites peuvent être mesurées avec une précision de l'ordre de $10 \mu \mathrm{m}$ (environ un dixième de l'épaisseur d'un cheveu). Elles sont dues aux variations locales du champ de gravité terrestre. Ces variations de gravité dépendent, entre autres, des variations de masse d'eau dans toute la colonne verticale du sol. Des algorithmes d'inversion permettent donc de fournir des estimations de ces variations de masse d'eau à l'échelle globale, au pas de temps mensuel et avec une résolution Forschungsanstalt für Luft und spatiale de l'ordre de $300 \mathrm{~km}$. La durée de vie initiale de la mission était de 5 ans. Cependant, plus de 14 ans après le lancement, elle fournit encore des données exploitables, bien qu'il y ait plusieurs données manquantes depuis 2011 suite à des problèmes de vieillissement des satellites. Son successeur, Grace Follow-On, est prévu, au mieux, pour 2017

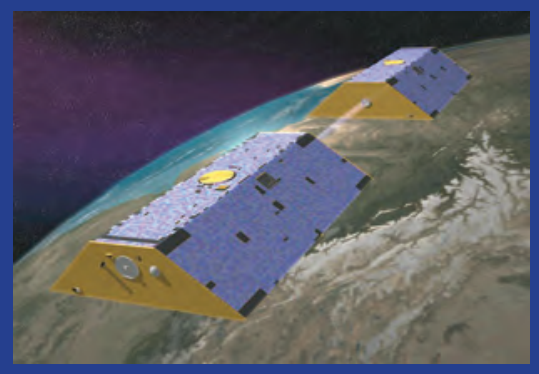

Vue d'artiste de la mission Grace. Crédit Nasa/JPL-Caltech.

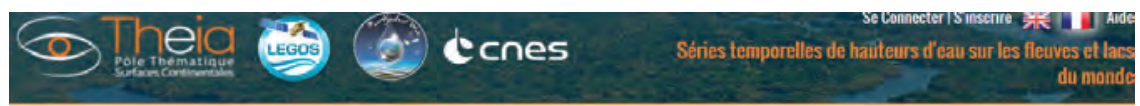

Lac Nasse

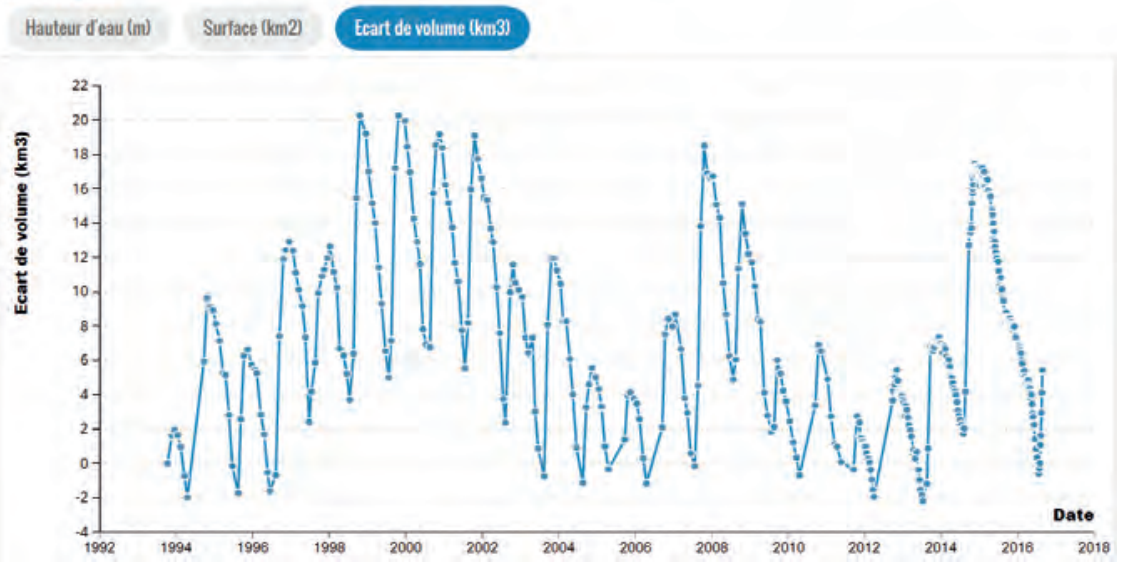

Figure 4. Variation de volume (en $\mathrm{km}^{3}$ ) du lac Nasser (Égypte) par rapport au volume à la première date d'observation, obtenue par combinaison de cote d'eau altimétrique et d'images optiques. Source : http://hydroweb.theia-land.fr/ et Crétaux et al. (2011).

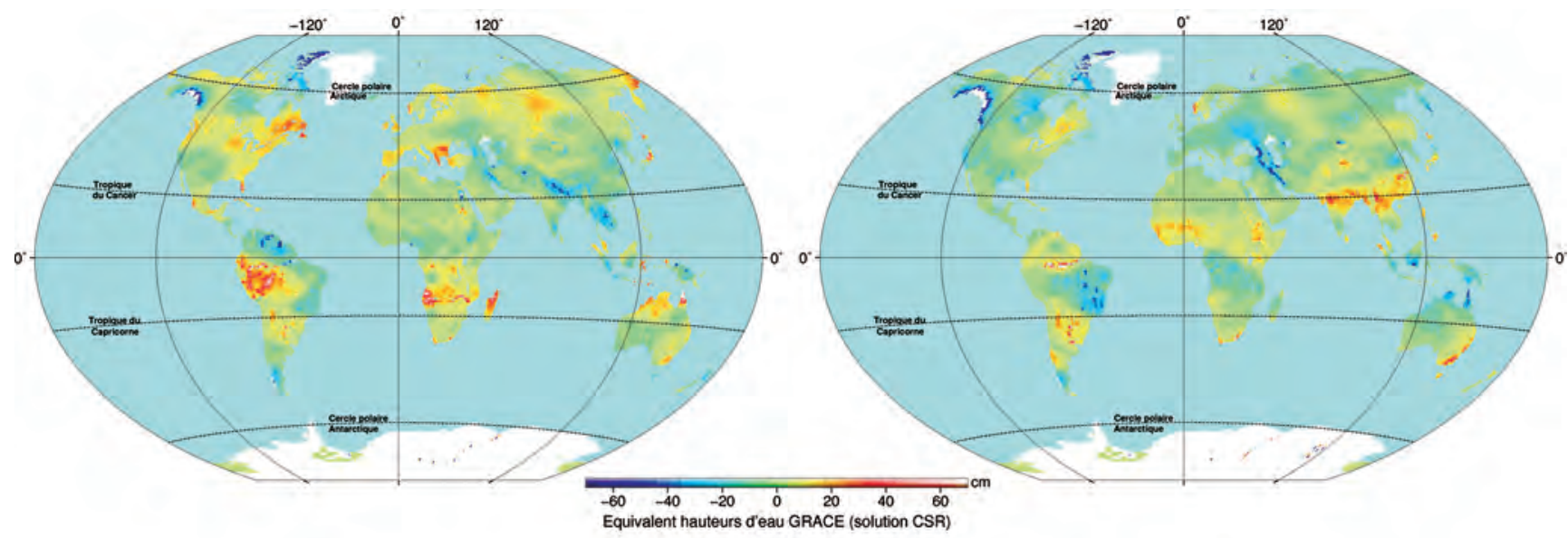

Figure 5. Variations de la hauteur d'eau totale (en cm) par rapport à la moyenne 2004-2009, estimées à partir des données Grace par le Center for Space Research (CSR) pour février 2015 (à gauche) et août 2015 (à droite). Ces variations ont été obtenues à partir des données Grace Tellus Land (http://grace.jpl.nasa.gov/data/get-data/monthly-mass-grids-land/). L'Antarctique et le Groenland sont en blanc, car ce produit n'est pas adapté pour les calottes (un produit Grace spécifique est disponible sur le site Grace Tellus). 
eau dans la végétation, eau contenue dans le manteau neigeux et dans les glaciers et calottes polaires, humidité du sol...) jusqu'à la roche mère (zone non saturée, aquifères de surface et aquifères profonds). Cette mission a permis de gros progrès dans l'étude du bilan hydrologique à l'échelle du bassin, car elle fournit une quantité qui ne peut être estimée in situ. En estimant les variations de masse d'eau de surface (en utilisant des modèles, des données in situ et les mesures des missions décrites précédemment) et en les soustrayant aux mesures Grace, il est possible d'avoir une estimation de la somme des variations de volume des aquifères de surface et profonds, qui ne peuvent être mesurées directement par satellite. Évidemment, ces estimations ont des incertitudes importantes, puisqu'elles incluent les erreurs d'observation Grace, ainsi que celles des données utilisées pour estimer les variations de volume des eaux de surface.

En ce qui concerne la qualité de l'eau, les missions optiques permettent d'estimer les flux sédimentaires et la concentration en chlorophylle des eaux, ne serait-ce qu'en surface (Martinez, 2015). Ces mesures fournissent de précieuses informations pour l'étude des processus d'érosion (naturels ou d'origine anthropique) et le suivi du phytoplancton à l'échelle du bassinversant, complétant les mesures in situ lorsqu'elles existent.

Cette présentation des données spatiales existantes pour l'hydrologie continentale a permis de donner un aperçu de la diversité des mesures disponibles, mais il faut également souligner la grande variabilité de leurs échelles spatio-temporelles, comme le montre le tableau 1. Combiner de multiples observations spatiales peut donc s'avérer difficile.

\section{Apport pour la grande échelle : meilleure connaissance du bilan d'eau}

Les observations satellitaires présentées précédemment ont permis d'améliorer notre connaissance du cycle de l'eau continental à grande échelle. Dans cette partie, nous présentons l'apport de ces données pour l'étude du cycle de l'eau à l'échelle d'un bassin fluvial, via le bilan hydrologique. Ce bilan correspond à l'inventaire des quantités d'eau reçues et perdues aux interfaces avec l'atmosphère et l'océan, à l'échelle de tout le bassin et sur une certaine période de temps. La somme de ces flux d'eau est égale à la variation de volume d'eau sur tout le bassin pendant ce même intervalle de temps. Ce bilan hydrologique à l'échelle du bassin fluvial peut être traduit par l'équation suivante :

$$
\Delta S / \Delta t=P-E-Q
$$

où $\Delta S / \Delta t$ est la variation du volume d'eau dans tous les compartiments (eau de surface, eau dans le sol et les aquifères) et sur tout le bassin pendant la période de temps considérée, divisée par cette période de temps.

$P$ correspond au flux de précipitations sous forme liquide (pluie) ou solide (neige, grêle) reçu à la surface de tout le bassin, en moyenne sur la période de temps considérée.

$E$ correspond au flux d'évapotranspiration, c'est-à-dire le flux d'eau «perdue » par le bassin et transférée vers l'atmosphère, via l'évaporation des surfaces continentales et la transpiration du couvert végétal. Cette valeur est considérée comme positive et est donc soustraite à l'eau reçue par le bassin via les précipitations $(P)$.

$Q$ correspond au débit sortant, soit la quantité d'eau s'écoulant par unité de temps hors du bassin à l'embouchure du réseau fluvial (ou échangé par les aquifères hors du bassin).

Ce bilan permet d'estimer les échanges entre la surface continentale d'une part et l'atmosphère et l'océan d'autre part, et ainsi de quantifier les évolutions de volume d'eau stockée dans le bassin, ce qui permet in fine de comprendre les causes et les conséquences de sécheresse, d'inondation et les ressources en eau disponibles dans le bassin.

Pour réaliser ce bilan, trois types de données sont principalement utilisés : les données in situ, les données de télédétection (satellitaires et aéroportées) et les modèles. Pour le bilan hydrologique, les modèles utilisés sont des modèles de surfaces continentales qui font un bilan de masse d'eau et d'énergie sur la colonne de sol. Ces modèles simulent aussi, de plus en plus fréquemment, le réseau fluvial de manière explicite via un modèle de routage. Ils sont donc souvent utilisés en combinaison avec les données satellitaires et/ou des mesures in situ.
Les données satellitaires présentées plus haut permettent d'estimer principalement les variations de volume d'eau $(\mathrm{d} S / \Delta t)$ dans différents compartiments du bassin ou dans sa totalité (eaux à surface libre, humidité du sol, neige, aquifères).

Les précipitations $(P)$ peuvent provenir de jeux de données in situ interpolées, de données de télédétection au sol ou depuis l'espace, de modèles ou bien d'une combinaison de ces approches. À grande échelle, les mesures de la constellation de satellites GPM (Global Precipitation Mission) se sont avérées être une source de données irremplaçable au vu du nombre important de bassins peu ou pas jaugés.

L'évapotranspiration $(E)$, quant à elle, reste difficile à estimer. Plusieurs études ont porté sur cette question et quelques réponses ont été apportées, mais jamais de façon totalement satisfaisante. De plus, peu de mesures in situ sont disponibles à grande échelle. Seules les mesures satellitaires et les modèles hydrologiques permettent d'en fournir une estimation plus ou moins cohérente et sont surtout liées à notre connaissance du milieu (état de ses variables) et de ses compartiments (tous les couverts ne transpirent pas de la même façon et cette transpiration est de toute façon pilotée par les forçages et l'eau disponible). De nombreux algorithmes ont été développés pour l'estimer à partir de données optiques (par exemple pour Landsat ou l'instrument Modis) et ont été validés localement par comparaison avec des mesures in situ. Les mesures dans l'infrarouge thermique sont plus adéquates pour suivre l'évapotranspiration réelle, mais ne sont malheureusement pas encore disponibles à la bonne résolution spatio-temporelle. De futures missions observant dans l'infrarouge thermique devraient aider à répondre à cette question.

Le débit sortant $(Q)$ est, le plus couramment, estimé à partir de données in situ et/ou de modèles. Cependant, ce débit n'est pas toujours disponible ou rendu public par les pays exploitant les stations de jaugeage, notamment pour les années récentes (c'est typiquement le cas en Sibérie ou pour certains pays d'Asie du Sud-Est ou d'Afrique). Des techniques fondées sur l'altimétrie nadir radar, sur des capteurs optiques ou bien sur des radiomètres permettent d'estimer ces débits en utilisant des données in situ sur une période commune. 
Certaines approches ont permis d'estimer ce bilan hydrologique en utilisant seulement des produits satellitaires (qui peuvent être corrigés en utilisant des réseaux in situ), comme par exemple Munier et al. (2014) sur le bassin du Mississippi. Ce type d'approche apporte des résultats satisfaisants à l'échelle d'un grand bassin et apporte des informations très utiles pour des bassins peu jaugés (le Mississippi ayant été utilisé comme site de validation) ; cependant, la seule utilisation de produits satellitaires entraîne de fortes incertitudes sur le bilan estimé.

Une autre approche assez répandue, consiste à utiliser ces produits satellitaires pour valider et/ou « corriger » les modèles hydrologiques. Les données spatiales ont été dans un premier temps (et sont toujours) utilisées pour valider les modèles globaux de surfaces continentales, du fait de leur vaste couverture spatiale. Ces mêmes données sont aussi de plus en plus utilisées pour étalonner certains paramètres de ces modèles, au même titre que les mesures in situ. Ces étalonnages et validations des modèles de surfaces continentales sont d'autant plus nécessaires que ces modèles sont couplés avec des modèles atmosphériques et océaniques pour simuler l'impact du changement climatique. Les données spatiales contribuent donc indirectement à l'amélioration de ces simulations. Enfin, depuis quelques années, les données spatiales (notamment l'humidité du sol, les étendues de neige, les données Grace et plus récemment les données altimétriques) sont de plus en plus assimilées dans les modèles hydrologiques globaux pour améliorer les estimations de variations de stock d'eau et ainsi fournir des réanalyses sur plusieurs années des variations de ces stocks (un exemple de ce type d'approche est le Land Data Assimilation System ${ }^{3}$ ). L'inconvénient de l'assimilation séquentielle est bien souvent la non-conservation de la masse aux temps d'assimilation, ce qui peut entraîner une non-fermeture du bilan hydrologique $(\Delta S / \Delta t$ n'est plus égal à $P-E-Q$ ).

L'ensemble de ces techniques (données spatiales seules, combinées avec des données in situ et leur assimilation dans des modèles hydrologiques) ont permis une meilleure compréhension et une vision synoptique de l'évolution de la distribution de zones en eau et de leur dynamique depuis les dernières décennies. Elles ont aussi permis de montrer leur dépendance et leur rétroaction avec les variations de l'état de l'atmosphère à grande échelle (corrélation avec les principaux indices climatiques, notamment ceux caractérisant le phénomène El Niño ou l'oscillation arctique...). Elles ont donc joué un rôle important dans la connaissance actuelle des ressources en eau à l'échelle des grands bassins fluviaux et du cycle de l'eau en général. Mais elles permettent aussi une vision des processus ayant lieu à méso-échelle ou à des échelles encore plus locales. Elles sont donc utilisées pour étudier des tronçons de rivière ou de fleuve, l'évolution de leur plaine d'inondation, les lacs ou l'impact des réservoirs, ce qui permet des applications plus opérationnelles.

\section{Apport pour les échelles régionales et locales, vers l'applicatif}

Les données satellitaires présentées précédemment ont permis aussi d'étudier, à différentes échelles spatiales, la dynamique des zones d'eau pendant des événements extrêmes (sécheresses, crues), la périodicité de ceux-ci, leur lien avec le climat et leurs interactions avec les sociétés humaines. Dans ce cadre, il est important de mentionner la volonté des principales agences spatiales, depuis 1999, de mettre à disposition les données de leurs satellites (notamment les images optiques) pour aider les secours, les agences gouvernementales et les organisations non gouvernementales lors de catastrophes naturelles ou d'origine humaine, via la charte internationale «Espace et catastrophes majeures ${ }^{4} » . \mathrm{La}$ $500^{\mathrm{e}}$ activation de cette charte a eu lieu le $1^{\text {er }}$ août 2016 lors d'inondations importantes au Bangladesh.

La tendance actuelle vise aussi à intégrer des données spatiales dans des systèmes de prévision de crues ou de sécheresses en complément ou bien au sein même de systèmes existants. On peut par exemple citer le système d'estimation de débit en temps quasi réel du Dartmouth Flood Observatory ${ }^{5}$ pour certains fleuves mondiaux, qui utilise des observations dans les spectres du visible, de l'infrarouge et des micro-ondes. On peut aussi citer le système de prévision du centre de prévision et d'alerte d'inondation du Bangladesh (Flood Forecasting and Warning Center ${ }^{6}$, agence gouvernementale) qui intègre des données d'altimétrie nadir depuis 2014 .

Les masques d'eau dérivés des données spatiales ont permis de montrer une évolution dans la distribution des lacs en Arctique (la région du globe contenant le plus de lacs) depuis les années 1970, en lien avec l'évolution du climat. Combinés avec l'altimétrie nadir, ces masques ont également permis de suivre sur près de 20 ans des variations de volume de lacs du plateau tibétain et de leur lien avec la variabilité climatique (Crétaux et al., 2016). Ils ont de plus permis le suivi du déplacement du lit mineur de grands fleuves, pour mettre à jour les cartes du chevelu des fleuves, pour ne citer que quelques exemples.

Les données spatiales citées dans cet article sont, en général, téléchargeables sur le site des agences spatiales ou de leurs partenaires. Devant la multiplication des données et des plateformes de téléchargement, certains sites ont été développés pour regrouper plusieurs types de données. Pour les surfaces continentales, la plupart des mesures des satellites de la Nasa (dont les données Landsat et Modis) peuvent être téléchargées directement sur le site Earth Explorer ${ }^{7}$ de l'USGS. Le pôle thématique surfaces continentales Théia ${ }^{8}$, soutenu par plusieurs agences et centres de recherches publics français, dont le Centre national d'études spatiales (Cnes), met à disposition de la communauté scientifique et des politiques publiques un catalogue de produits spatiaux très fourni (comme des images optiques corrigées des effets atmosphériques, des cotes d'eau estimées par altimétrie radar, des cartes d'occupation du sol, des indices de végétation...). Les données des missions Sentinel de l'ESA sont disponibles sur le portail Copernicus Open Access Hub9 Les mesures Smos, quant à elles, peuvent être téléchargées sur le site CATDS (Centre aval de traitement des données Smos $\left.{ }^{10}\right)$. Enfin, les données Grace

3. http://ldas.gsfc.nasa.gov/gldas/

4. https://www.disasterscharter.org/fr/web/guest

5. http://floodobservatory.colorado.edu/Discharge Access.html

6. http://ffwc.gov.bd/index.php/hydrograph/jason-

2-satellite-based-forecast

7. http://earthexplorer.usgs.gov/

8. https://www.theia-land.fr/fr

9. https://scihub.copernicus.eu/

10. https://www.catds.fr/Products/Productsaccess 
traitées par plusieurs centres de recherche, notamment pour les surfaces continentales, sont disponibles sur le site Grace Tellus ${ }^{11}$ et sur le site Grace/ Lageos $^{12}$. Il ne s'agit évidemment pas d'un inventaire exhaustif des portails de téléchargement, mais ces sites permettent d'avoir accès aux principales données satellitaires citées dans cet article.

\section{Conclusions et perspectives}

Cet aperçu de l'utilisation des observations spatiales pour l'hydrologie continentale illustre le vaste spectre de données utilisables et de champs d'application, qui donnent une vision synoptique des ressources en eau disponibles sur les continents et de leur variabilité. Ces données ne remplaceront pas les mesures in situ, mais en sont un complément important, notamment pour les bassins peu ou pas jaugés et pour le cas des bassins transfrontaliers.

Dans les prochaines années, de nouvelles missions vont être lancées pour continuer les séries temporelles acquises, mais aussi pour fournir de nouvelles données qui devraient encore améliorer notre connaissance du cycle de l'eau continental. On peut notamment citer la continuité du programme Sentinel de l'ESA, avec des lancements de nouveaux satellites au moins jusqu'en 2020 qui vont compléter les satellites déjà lancés et offrir une gamme de données sans précédent. L'observation des variations de volume total d'eau sera poursuivie grâce à la mission Grace-FO (Grace Follow-On), dont le lancement est prévu pour 2017. Pour les eaux à surface libre, la future mission Swot (de la Nasa, du Cnes, de l'Agence spatiale canadienne et de la UK Space Agency) devrait être lancée d'ici 2021. Elle fournira, pour la première fois, des images des cotes d'eau sur pratiquement toutes les zones d'eau à surface libre, ce qui permettra un suivi sans précédent des flux et des variations de masse d'eau des fleuves, rivières, lacs et réservoirs du monde entier. Ces nouvelles missions vont permettre de nombreuses observations avec des résolutions spatiales et temporelles plus précises que par le passé.

Cependant, il reste encore de nombreux défis à relever dans le cadre de l'observation des continents depuis l'espace. Le premier est d'assurer la pérennité des observations, afin d'obtenir des observations cohérentes dans le temps, sans interruption de mesure, sur une longue période de temps (plusieurs décennies), pour pouvoir réaliser des études climatiques. Le transfert vers les gestionnaires, les décideurs politiques et les industriels des avancées de la recherche est aussi un enjeu majeur afin de faire bénéficier l'ensemble de la société des avancées récentes de la recherche dans ce domaine. D'un point de vue scientifique, l'amélioration de l'observation ou l'observation directe de certains compartiments du cycle de l'eau continentale comme les aquifères de surface et profonds, du débit des fleuves ou de l'évapotranspiration serait d'un grand bénéfice pour améliorer notre connaissance de l'hydrologie continentale. De même, le suivi de la qualité des eaux (température, suivi de matières organique, polluants...) est encore difficilement réalisable sur toute la colonne d'eau avec les données disponibles à l'heure actuelle.

L'impact anthropique direct et du changement climatique sur les ressources en eau est de plus en plus étudié et va se poursuivre dans les années à venir. Dans ce cadre, il est certain que les mesures spatiales vont continuer à jouer un rôle important.

11. http://grace.jpl.nasa.gov/

12. http://grgs.obs-mip.fr/grace

\section{Bibliographie}

Brocca L., Pellarin T., Crow W.T., Ciabatta L., Massari C., Ryu D., Su C.-H., Rüdiger C., Kerr Y.H., 2016. Rainfall estimation by inverting SMOS soil moisture estimates: a comparison of different methods over Australia. J. Geophys. Res. Atmos., 121, 12062-12079. doi: 10.1002/2016JD025382

Crétaux J.-F., Jelinski W., Calmant S., Kouraev A., Vuglinski V., Berge-Nguyen M., Gennero M.-C., Nino F., Abarca-del-Río R., Cazenave A., Maisongrande P., 2011. SOLS: A lake database to monitor in the near real time water level and storage variations from remote sensing data. Adv. Space Res., 47, $1497-1507$.

Crétaux J.-F., Abarca-del-Río R., Bergé-Nguyen M., Arsen A., Drolon V., Clos G., Maisongrande P., 2016. Lake volume monitoring from space. Surv. Geophys., 37, 269-305. doi: 10.1007/s10712-016-9362-6

Entekhabi D., Njoku E.G., O’Neill P.E., Kellogg K.H., Crow W.T., Edelstein W.N., Entin J.K., Goodman S.D., Jackson T.J., Johnson J., Kimball J., Piepmeier J.R., Koster R.D., Martin N., McDonald K.C., Moghaddam M., Moran S., Reichle R., Shi J.C., Spencer M.W., Thurman S.W., Tsang L., Zyl J.V., 2010. The soil moisture active passive (SMAP) mission. Proceedings of the IEEE, 98, 704-716.

Kerr Y.H., 2007. Soil moisture from space: Where are we? Hydrogeol. J., 15, 117-120.

Kerr Y.H. et al., 2016. Overview of SMOS performance in terms of global soil moisture monitoring after six years in operation. Remote Sens. Environ., 180, 40-63.

Lettenmaier D.P., Alsdorf D., Dozier J., Huffman G.J., Pan M., Wood E.F., 2015. Inroads of remote sensing into hydrologic science during the WRR era. Water Resour. Res., 51, 7309-7342. doi: 10.1002/2015WR017616

Martinez J.-M., 2015. Mesure de la qualité des eaux par satellite. In : Euzen A., Jeandel C., Mosseri R., eds, L'eau à découvert. CNRS Éditions, Paris, $216-217$.

Merlin 0., Jose Escorihuela M., Aran Mayoral M., Hagolle 0., Al Bitar A., Kerr Y.H., 2013. Self-calibrated evaporation-based disaggregation of SMOS soil moisture: An evaluation study at $3 \mathrm{~km}$ and $100 \mathrm{~m}$ resolution in Catalunya, Spain. Remote Sens. Environ., 130, 25-38.

Munier S., Aires F., Schlaffer S., Prigent C., Papa F., Maisongrande P., Pan M., 2014. Combining data sets of satellite-retrieved products for basin-scale water balance study: 2. Evaluation on the Mississippi Basin and closure correction model. J. Geophys. Res. Atmos., 119, 12100-12116. doi: 10.1002/2014JD021953

Rabatel A., Berthier E., Arnaud Y., Dedieu J.-P., Dumont M., Gascoin S., Gay M., Guerreiro K., Karbou F., Kouraev A., Picard G., Rémy F., Sirguey P., Trouvé E., Zakharova E., 2017. Télédétection satellitaire des surfaces enneigées et englacées. La Météorologie, 97, 78-85.

Rautiainen K., Parkkinen T., Lemmetyinen J., Schwank M., Wiesmann A., Ikonen J., Derksen C., Davydov S., Davydova A., Boike J., Langer M., Drusch M., Pulliainen J., 2016. SM0S prototype algorithm for detecting autumn soil freezing. Remote Sens. Environ., 180, 346-360.

Schwank M., Matzler C., Wiesmann A., Wegmuller U., Pulliainen J., Lemmetyinen J., Rautiainen K., Derksen C., Toose P., Drusch M., 2015. Snow density and ground permittivity retrieved from L-band radiometry: a synthetic analysis. IEEE J. Selected Topics Applied Earth Obs. Remote Sens., 8, 3833-3845.

Waldteufel P., Kerr Y.H., Boutin J., 2012. SMOS vole au-dessus de nos têtes. La Météorologie, 76, 34-43. 